\title{
Quality of life of patients with Psoriasis in Alexandria- Egypt
}

\author{
Dr. Amany Youssef Sharaf, Dr. Alyaa Farouk Abd El-Fattah Ibrahim \\ Lecturer of Medical-Surgical Nursing, Faculty of Nursing, Alexandria University. Egypt \\ Lecturer of Community Health Nursing, Faculty of Nursing, Alexandria University. Egypt \\ Assistant Professor of Community Health Nursing, College of Nursing, King Saud bin Abdul-Aziz University for \\ Health Sciences. KSA
}

\begin{abstract}
:
Background: Psoriasis is an obvious systemic problem that affects somatic, psychologic and social aspects of life. Therefore, quality of life (QoL) of psoriasis patients is often significantly impaired.

Objectives: The present study aimed to assess the quality of life of patients with psoriasis and to compare the impact of different grades of disease severity on patients' quality of life domains.

Setting: Dermatology Outpatient Clinic of Alexandria Main University Hospital.

Patients and Methods: This is a descriptive study. Data were collected from 110 patients attending the prementioned health setting. A socio demographic, clinical characteristics structured questionnaire, and the Dermatological Quality of Life Scale DQOLS, were utilized for data collection. Each patient was interviewed individually after brief explanation of the aims of the study. The interview took about 30 minutes for each patient.
\end{abstract}

Results: Significant relationships were noted between quality of life of patients' activities of daily living and physical activities percent scores and severity of psoriasis $\left(\chi^{2}=68.723 \& P<0.001\right) .,\left(\chi^{2}=32.440 \& P<\right.$ 0.001 ). Working conditions, financial status, social status were significantly related with the severity of the disease. The majority of the studied patients had poor quality of life in relation to physical activity, social relationships, working conditions, and financial status $(62.7 \%, 56.4 \%, 52.7 \%$ \& $99.1 \%$, respectively.

Conclusion: Psoriasis has negative impact on the majority of patients' quality of life and there were positive associations between disease severity and patients' poor quality of life

Recommendations : Develop rehabilitation programs to enhance psoriasis patient's self management and quality of life.

Keywords: Psoriasis, quality of life, Psoriasis disability

\section{Introduction}

Psoriasis is a chronic non-communicable skin disease characterized by red papules and plaques with a silver colored scale in a spontaneous remission and relapse course with no clear cause or cure ${ }^{(1,2)}$. Although, there is evidence for genetic predisposition; the role of the immune system in psoriasis causation is being studied $^{(3)}$. As, psoriasis is suggested to be an autoimmune disease; no autoantigen that could be responsible has been defined yet. External and internal triggers, including mild trauma, sunburn, infections, smoking, high alcohol consumption, seasonal and hormonal changes, systemic drugs and stress might be responsible for Psoriasis provocation ${ }^{(4)}$.

Psoriasis affects people of all ages, its onset can be at any age with peak at 50-69 years, both males and females are equally affected in all countries ${ }^{(5)}$. It has become a significant health problem in modern society affecting about $0.09 \%-11.43 \%$ of the world population making psoriasis a serious global problem with at least 100 million individuals affected worldwide ${ }^{(5)}$. Recent review also suggests that globally the prevalence of psoriasis may be increasing ${ }^{(6)}$.

Roughly, Psoriasis affects 3 percent of the US population and about 250,000 new cases develop the disease every year ${ }^{(7,8)}$. In most developed countries, the prevalence is between 1.5 and $5 \%^{(9)}$. In Egypt, Few epidemiological surveys have been carried out to determine the prevalence of skin diseases in the Egyptian population ${ }^{(10)}$.

Since Psoriasis is an obvious systemic problem that affects somatic, psychologic and social aspects of the life, quality of life (QoL) of psoriasis patients is often significantly altered ${ }^{(11,12)}$. Disfiguration, disability and marked loss of productivity are common challenges for people with psoriasis. Evidence also reported an association between psoriasis and severe physical conditions such as psoriatic arthritis, cardiovascular disease, metabolic syndrome, and Crohn's disease. In addition, life expectancy is reduced by several years in individuals with severe disease, mainly caused by increased risk of heart disease ${ }^{(13-16)}$

The significant cost to mental well-being, such as higher rates of depression, negatively impacts individuals and society ${ }^{(17,18)}$. Social exclusion, discrimination and stigma are psychologically devastating for individuals suffering from psoriasis and their families. Psoriasis affects relationships at home, school and work 
as well as sexual relationships and thus reduce QoL and adds to the social and psychological $\operatorname{strain}^{(19,20)}$. Moreover, the chronic recurrent nature of the disease, the erythema and scaliness lead to the hopelessness of complete cure to its treatment and have a great impact on patient self-esteem and confidence and reported cases of suicidal attacks ${ }^{(21-23)}$.

Psoriasis management is another challenge affecting the patient's quality of life as regards; the way of application, cost, side effects, access to health care and beneficial results ${ }^{(24)}$. Additionally, functional impairment, lost opportunities in professional life and elevated economic burden for treatment expenses can add to significant socioeconomic burden in the affected patients ${ }^{(25)}$. Care for patients with psoriasis requires not only treating physical manifestations, but it is also very important to identify and manage psychological conditions ${ }^{(26)}$.

As the primary goal of QoL is to ensure the high level of well being of people despite their disabilities, nurses have a positive role in patient education, and planning nursing interventions aiming at minimizing the consequences of psoriasis, and improving patients' quality of life through effective management ${ }^{\text {(27) }}$. In addition, nurses are in a unique position to address the multidimensional impact of psoriasis on patients' QoL by allowing them to verbalize fears and concerns, meeting with significant others, teaching patients about the disease process, assisting patients to participate in their usual activities, encouraging patients to comply with their treatment and suggest lifestyle modifications that will potentially improve patients' quality of life ${ }^{(28)}$.

There is a great challenge to improve the quality of life in psoriasis patients. Specific therapeutic and psychologic strategies are needed to improve the negative impact of the disease on the general health. As, literature review revealed limited studies assessing the impact of Psoriasis on patients' quality of life in Egypt, lack of data necessitates the design of this study.

Aims of the study

- $\quad$ To assess the QoL of psoriasis patients in Alexandria.

- $\quad$ To compare the impact of different grades of disease severity on patients' QoL domains.

Research questions

- How does Psoriasis affect QoL of the affected patients?

$\circ \quad$ What is the impact of different grades of psoriasis disease severity on patient's QoL?

\section{Materials and method}

\section{Materials}

Design: A descriptive research design was used for the purpose of study.

Settings: The Dermatology Outpatient Clinic of Alexandria Main University, Hospital, Alexandria, Egypt.

Subjects: The study comprised a purposive sample of 110 adult Psoriasis patients attending the pre-mentioned health setting and meeting the following criteria:

- Age: 20- 60 years old

- Had a definite diagnosis of psoriasis confirmed by the dermatologists.

- Absence of uncontrollable illnesses (Hypertension, Heart diseases, ....etc) or any other dermatology diseases.

Sample size calculation: Epi info -7 programs was used to estimate the sample size using the following parameters:

1- Population size $=150$

2- Expected frequency $=50 \%$

3 -Acceptable error $=5 \%$

4-Confidence co efficient $=95 \%$

5-Minimum sample size $=108$ patients

Tools: based on an extensive review of related literature; two tools were used for the purpose of data collection.

Tool I: Socio demographic and clinical characteristics structured questionnaire: It includes questions related to the socio demographic characteristics of the studied patients (area of residence, age, sex, education, occupation, social status, family type, income) and questions related to the clinical characteristics of the studied patients ( severity of psoriasis, area covered or exposed \& onset of the disease).

Tool II: The Dermatological Quality of Life Scale (DQOLS): This scale was adapted and translated into Arabic by the researchers from Morgan et al. $(1997)^{(29)}$. The adapted questionnaire comprised six domains with a total of 26 questions. Affected activities of daily livings as eating \& sleep (4 questions), affected physical activity (5 questions), social relationships items (5 questions), psychological and sexual feeling items (6 questions), affected working items (2 questions), and affected financial items (4 questions). Patients were asked to respond on a 5 point likert scale representing grades from "not at all" to "all the time. One score was given to 
the "not at all" answer, 2 for "very seldom", 3 for "sometimes", 4 for "nearly always", and 5 for "always". Missing answers were deleted from the total score. Answers of the studied patients were recorded, scored, then summed together. The total score was categorized into three levels as follows:-

○ Poor quality of life: less than $50 \%$

- Fair quality of life: $50 \%$ to less than $75 \%$

- Good quality of life: equal or more than $75 \%$

\section{Method}

Ethical considerations: The current study was accepted by the Research Institutional Review Board of the Faculty of Nursing, Alexandria University.

- Permission to carry out the study was obtained from the directors and the responsible authorities of the identified setting after explaining aims of the study.

- Psoriasis patients (study participants) were approached and given a covering letter preceding the questionnaire which included a written description of the purpose and nature of the study and a written consent to participate in the study was obtained from them. For illiterate patients, an oral explanation and the covering letter was read to them.

- Assurance of anonymity was addressed prior to the request for participation, thus all information remained confidential. In addition, the studied patients were reassured that their participation in the study is voluntary. All patients were informed that they could withdraw from the study at any time if they wish not to participate.

- Confidentiality was maintained by data coding to eliminate identifying data with personal information.

- Tool I was developed and tool II was adapted, and translated into Arabic after extensive review of relevant and current literature.

- Content and construct validity, as well as the domains representative of the developed instruments were ascertained by a jury of 5 experts in the fields of medical surgical nursing, community health nursing and dermatology. The necessary modifications were modified accordingly.

- Internal consistency was used in ascertaining reliability of the instrument using Cronbach Alpha Coefficient Test (0.942), which means that the data were reliable.

\section{Pilot study}

Tools were pre-tested on $10 \%$ of the studied patients who were not included in the further study sample. Data obtained from the pilot study were analyzed, some questions were restated and some items were added accordingly.

\section{Data collection:}

- Every patient was interviewed individually after brief explanation of the aims of the study. The interview took around 30 minutes each.

- Data was collected over a period of 4 months starting from January to April 2016.

\section{Statistical analysis:}

After data collection, it was coded and transferred into a specially designed format to be suitable for computer feeding. Following data entry, checking and verification process were carried out to avoid any error during data entry.

Statistical analysis was performed using Statistical package for Social Sciences (SPSS version 20) and data tabulated. The level of significance selected for this study was p equal to or less than 0.05 .

\section{The following statistical measures were used:}

- Descriptive measures included: frequency and percentages used for describing and summarizing variables. Arithmetic means, standard deviation, range (maximum and minimum) were used as measures of control tendency and dispersion respectively, for normally distributed quantitative data.

- Statistical tests included:

1- Chi-square test: for categorical variables, to compare between different groups

2 - Monte Carlo correction: for correction for chi-square when more than $20 \%$ of the cells have expected count less than 5

3 - F-test (ANOVA): for normally quantitative variables, to compare between more than two groups

\section{Results}

Among the 118 psoriasis patients who were initially approached, 110 agreed to participate in the study giving a response rate of $93.2 \%$. Lack of time, disease stigma, and absence of interest, were mainly the reasons 
for refusal to participate in the study. Demographic and clinical characteristics are shown in (Table 1). Nearly three quarters $(75.2 \%)$ of the included psoriasis patients reside urban areas, their age ranged between 30 and 60 years with a mean age of $51.43 \pm 8.57$ years. Males, illiterate patients represented slightly more than half of the studied sample $(55.5 \%, 54.5 \%)$ respectively. Also, the majority of the studied patients were unemployed, married, and had monthly income less than 1000 pounds.

Table (1): Distribution of the studied patients according to their socio-demographic and clinical characteristics $(\mathrm{n}=110)$

\begin{tabular}{|c|c|c|}
\hline Socio-demographic \& Clinical characteristics & No. & Percent (\%) \\
\hline \multicolumn{3}{|l|}{ Residence } \\
\hline Urban & 83 & 75.5 \\
\hline Rural & 27 & 24.5 \\
\hline \multicolumn{3}{|l|}{ Age (years) } \\
\hline $30>40$ & 22 & 20.0 \\
\hline $40>50$ & 17 & 15.5 \\
\hline $50-60$ & 71 & 64.5 \\
\hline Min-Max (Age) & \multicolumn{2}{|l|}{$30.0-60.0$} \\
\hline Mean \pm SD & \multicolumn{2}{|l|}{$51.43 \pm 8.57$} \\
\hline Median & \multicolumn{2}{|l|}{55.0} \\
\hline \multicolumn{3}{|l|}{ Sex } \\
\hline Male & 61 & 55.5 \\
\hline Female & 49 & 44.5 \\
\hline \multicolumn{3}{|l|}{ Education level } \\
\hline Illiterate & 60 & 54.5 \\
\hline Read and write & 19 & 17.3 \\
\hline High school or less & 24 & 21.8 \\
\hline University education or more & 7 & 6.4 \\
\hline \multicolumn{3}{|l|}{ Occupation } \\
\hline Clerical/Technical & 9 & 8.2 \\
\hline Manual & 49 & 44.5 \\
\hline Unemployed & 50 & 45.5 \\
\hline Retired & 2 & 1.8 \\
\hline \multicolumn{3}{|l|}{ Social status } \\
\hline Single & 3 & 2.7 \\
\hline Married & 95 & 86.4 \\
\hline Widow & 12 & 10.9 \\
\hline \multicolumn{3}{|l|}{ Family type } \\
\hline Nuclear & 98 & 89.1 \\
\hline Extended & 12 & 10.9 \\
\hline \multicolumn{3}{|l|}{ Monthly income (EGP per month) } \\
\hline$<1000$ & 69 & 62.7 \\
\hline $1000-2000$ & 38 & 34.5 \\
\hline$>2000$ & 3 & 2.7 \\
\hline \multicolumn{3}{|l|}{ Severity of psoriasis } \\
\hline Mild & 34 & 30.9 \\
\hline Moderate & 43 & 39.1 \\
\hline Severe & 33 & 30.0 \\
\hline \multicolumn{3}{|l|}{ Psoriasis area Covered } \\
\hline Exposed (visible) & 48 & 43.6 \\
\hline Covered (not visible) & 62 & 56.4 \\
\hline \multicolumn{3}{|l|}{ History of psoriasis } \\
\hline$<1$ year & 54 & 49.1 \\
\hline$>1$ year & 56 & 50.9 \\
\hline
\end{tabular}

Figure (1) illustrates that the majority of the studied patients had poor QoL in relation to physical activity, social relationships, working conditions, and financial status $(62.7 \%, 56.4 \%, 52.7 \%$ \& $99.1 \%$, respectively), while more than three quarters $(75.5 \%)$ of the participants had fair QoL in relation to activities of daily livings as eating and sleeping. On the other hand, the figure shows that slightly less than half $(48.2 \%)$ of the studied patients had good QoL in relation to psychosocial and sexual status. 


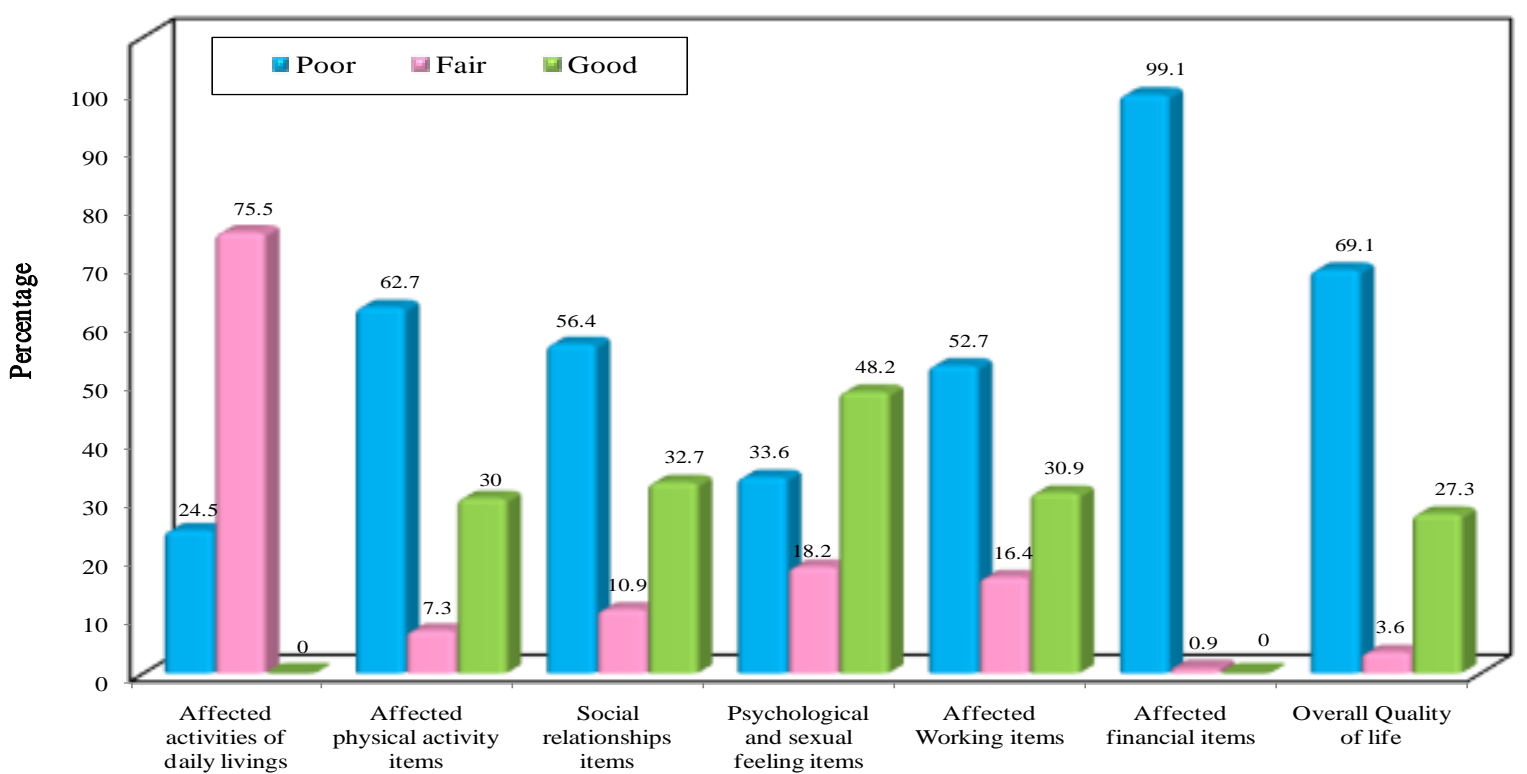

Figure (1): Distribution of the studied patients according to their percent score of psoriasis Quality of life Domains $(n=110)$

Table (2) Strong statistically significant relationships were detected between Psoriasis severity and patient's education, social status, family type and having the disease for more than one year $\chi^{2}=13.148 \& \mathrm{P}=0.030, \chi^{2}$ $=14.577 \& \mathrm{P}=0.001, \chi^{2}=8.801 \& \mathrm{P}=0.008 \& \chi^{2}=67.195 \& \mathrm{P}<0.001$, respectively. However, there was no statistical association between psoriasis severity and place of residence, age, sex, occupation and monthly income of the studied patients.

Table (2): Relationship between severity of psoriasis and socio-demographic and clinical characteristics of the studied patients

\begin{tabular}{|c|c|c|c|c|c|c|c|c|}
\hline \multirow{3}{*}{$\begin{array}{l}\text { Socio-demographic } \quad \& \quad \text { Clinical } \\
\text { Characteristics }\end{array}$} & \multicolumn{6}{|c|}{ Severity Of Psoriasis } & \multirow{3}{*}{$\begin{array}{l}\text { Test of } \\
\text { Sig. }\end{array}$} & \multirow{3}{*}{$\mathbf{P}$} \\
\hline & \multicolumn{2}{|l|}{$\begin{array}{l}\text { Mild } \\
(n=34)\end{array}$} & \multicolumn{2}{|c|}{$\begin{array}{l}\text { Moderate } \\
(\mathrm{n}=\mathbf{4 3})\end{array}$} & \multicolumn{2}{|c|}{$\begin{array}{l}\text { Severe } \\
(n=33)\end{array}$} & & \\
\hline & No & $\%$ & No & $\%$ & No & $\%$ & & \\
\hline \multicolumn{9}{|l|}{ Residence } \\
\hline Urban & 25 & 73.5 & 30 & 69.8 & 28 & 84.8 & \multirow{2}{*}{$\begin{array}{c}\chi^{2}= \\
2.391\end{array}$} & \multirow{2}{*}{0.303} \\
\hline Rural & 9 & 26.5 & 13 & 30.2 & 5 & 15.2 & & \\
\hline \multicolumn{9}{|l|}{ Age } \\
\hline $30>40$ & 7 & 20.6 & 6 & 14.0 & 9 & 27.3 & \multirow{3}{*}{$\begin{array}{c}\chi^{2}= \\
2.504\end{array}$} & \multirow{3}{*}{0.644} \\
\hline $40>50$ & 6 & 17.6 & 6 & 14.0 & 5 & 15.2 & & \\
\hline $50-60$ & 21 & 61.8 & 31 & 72.1 & 19 & 57.6 & & \\
\hline Min. - Max. & \multicolumn{2}{|l|}{$28.0-67.0$} & \multicolumn{2}{|c|}{$35.0-63.0$} & \multicolumn{2}{|c|}{$38.0-63.0$} & \multirow{4}{*}{$\begin{array}{c}\mathrm{F}= \\
1.699\end{array}$} & \multirow{3}{*}{0.188} \\
\hline Mean \pm SD. & $51.85 \pm 10.96$ & & \multicolumn{2}{|c|}{$53.28 \pm 7.38$} & \multicolumn{2}{|c|}{$49.48 \pm 8.39$} & & \\
\hline Median & \multicolumn{2}{|l|}{57.50} & 54. & & 53. & & & \\
\hline \multicolumn{8}{|l|}{ Sex } & \\
\hline Male & 16 & 47.1 & 24 & 55.8 & 21 & 63.6 & \multirow{2}{*}{$\begin{array}{c}\chi^{2}= \\
1.867\end{array}$} & \multirow{2}{*}{0.393} \\
\hline Female & 18 & 52.9 & 19 & 44.2 & 12 & 36.4 & & \\
\hline \multicolumn{9}{|l|}{ Education level } \\
\hline Illiterate & 21 & 61.8 & 28 & 65.1 & 11 & 33.3 & \multirow{4}{*}{$\begin{array}{c}\chi^{2}= \\
13.148^{*}\end{array}$} & \multirow{4}{*}{$\begin{array}{l}{ }^{\mathrm{MC}} \mathrm{p}= \\
0.030^{*}\end{array}$} \\
\hline Read and write & 7 & 20.6 & 7 & 16.3 & 5 & 15.2 & & \\
\hline High school or less & 5 & 14.7 & 7 & 16.3 & 12 & 36.4 & & \\
\hline University education & 1 & 2.9 & 1 & 2.3 & 5 & 15.2 & & \\
\hline \multicolumn{9}{|l|}{ Occupation } \\
\hline Clerical/Technical & 5 & 14.7 & 4 & 9.3 & 0 & 0.0 & \multirow{4}{*}{$\chi^{2}=9.013$} & \\
\hline Manual & 15 & 44.1 & 17 & 39.5 & 17 & 51.5 & & ${ }^{\mathrm{MC}} \mathrm{p}=$ \\
\hline Unemployed & 14 & 41.2 & 22 & 51.2 & 14 & 42.4 & & 0.116 \\
\hline Retired & 0 & 0.0 & 0 & 0.0 & 2 & 6.1 & & \\
\hline Social status & & & & & & & & \\
\hline Single & 0 & 0.0 & 0 & 0.0 & 3 & 9.1 & & \\
\hline Married & 31 & 91.2 & 42 & 97.7 & 22 & 66.7 & $\begin{array}{c}\chi^{2}= \\
\end{array}$ & $\mathrm{p}=$ \\
\hline Widow & 3 & 8.8 & 1 & 2.3 & 8 & 24.2 & & \\
\hline Family type & & & & & & & & \\
\hline
\end{tabular}


Quality of life of patients with Psoriasis in Alexandria- Egypt

\begin{tabular}{|c|c|c|c|c|c|c|c|c|}
\hline Nuclear & 31 & 91.2 & 34 & 79.1 & 33 & 100.0 & \multirow{2}{*}{$\begin{array}{c}\chi^{2}= \\
8.801^{*}\end{array}$} & \multirow{2}{*}{$\begin{array}{c}{ }^{\mathrm{MC}}= \\
0.008^{*}\end{array}$} \\
\hline Extended & 3 & 8.8 & 9 & 20.9 & 0 & 0.0 & & \\
\hline \multicolumn{9}{|l|}{ Monthly income } \\
\hline$<1000$ & 22 & 64.7 & 31 & 72.1 & 16 & 48.5 & \multirow{3}{*}{$\begin{array}{c}\chi^{2}= \\
7.232\end{array}$} & \multirow{3}{*}{0.080} \\
\hline $1000-2000$ & 10 & 29.4 & 11 & 25.6 & 17 & 51.5 & & \\
\hline$>2000$ & 2 & 5.9 & 1 & 2.3 & 0 & 0.0 & & \\
\hline $\begin{array}{l}\text { History of Psoriasis } \\
<1 \text { year } \\
>1 \text { year }\end{array}$ & $\begin{array}{l}34 \\
0\end{array}$ & $\begin{array}{l}100 \\
0.0\end{array}$ & $\begin{array}{l}20 \\
23\end{array}$ & $\begin{array}{l}46.5 \\
53.5\end{array}$ & $\begin{array}{l}0 \\
33\end{array}$ & $\begin{array}{l}0.0 \\
100\end{array}$ & $\begin{array}{c}\chi^{2}=67.19 \\
5^{*}\end{array}$ & $<0.001^{*}$ \\
\hline
\end{tabular}
$\chi^{2}$ : Chi square test
MC: Monte Carlo for Chi square test
F: F test (ANOVA)
*: Statistically significant at $\mathrm{p} \leq 0.05$

Table (3) presents high statistically significant relationships between severity of psoriasis and all domains of patients' quality of life. Patients with moderate to severe psoriasis had poor to fair quality of life, while patients with mild psoriasis had good quality of life in all domains, except the financial one, which was poor $\left(\chi^{2}=2.008\right.$ $\& \mathrm{P}=0.608)$.

Table (3): Relationship between quality of life scores and severity of psoriasis $(\mathrm{n}=110)$

\begin{tabular}{|c|c|c|c|c|c|c|c|c|}
\hline \multirow[t]{3}{*}{ quality of life domains scores } & \multicolumn{6}{|c|}{ severity of psoriasis } & \multirow{3}{*}{ Test of Sig. } & \multirow{3}{*}{$\mathbf{P}$} \\
\hline & \multicolumn{2}{|c|}{$\begin{array}{l}\text { Mild } \\
(n=34)\end{array}$} & \multicolumn{2}{|c|}{$\begin{array}{l}\text { Moderate } \\
(n=43)\end{array}$} & \multicolumn{2}{|c|}{$\begin{array}{l}\text { Severe } \\
(\mathbf{n}=33)\end{array}$} & & \\
\hline & No & $\%$ & No & $\%$ & No & $\%$ & & \\
\hline \multicolumn{9}{|l|}{ Daily Living activities } \\
\hline Poor quality & 0 & 0.0 & 12 & 27.9 & 15 & 45.5 & \multirow{3}{*}{$\begin{array}{c}\chi^{2}= \\
19.112^{*}\end{array}$} & \multirow{3}{*}{$<0.001^{*}$} \\
\hline Fair quality & 34 & 100.0 & 31 & 72.1 & 18 & 54.5 & & \\
\hline Good & 0 & 0.0 & 0 & 0.0 & 0 & 0.0 & & \\
\hline Mean \pm SD. & \multicolumn{2}{|c|}{$61.95 \pm 2.37$} & \multicolumn{2}{|c|}{$54.36 \pm 7.16$} & \multicolumn{2}{|c|}{$43.37 \pm 18.08$} & $\mathrm{~F}=24.423^{*}$ & $<0.001^{*}$ \\
\hline \multicolumn{9}{|l|}{ Physical Activity } \\
\hline Poor quality & 0 & 0.0 & 43 & 100.0 & 26 & 78.8 & \multirow{3}{*}{$\begin{array}{c}\chi^{2}= \\
128.422^{*}\end{array}$} & \multirow{3}{*}{${ }^{\mathrm{MC}} \mathrm{p}<0.001^{*}$} \\
\hline Fair quality & 1 & 2.9 & 0 & 0.0 & 7 & 21.2 & & \\
\hline Good & 33 & 97.1 & 0 & 0.0 & 0 & 0.0 & & \\
\hline Mean \pm SD. & \multicolumn{2}{|c|}{$96.47 \pm 8.92$} & \multicolumn{2}{|c|}{$26.05 \pm 4.16$} & \multicolumn{2}{|c|}{$17.42 \pm 21.87$} & $\mathrm{~F}=374.459^{*}$ & $<0.001^{*}$ \\
\hline \multicolumn{9}{|l|}{ Social status } \\
\hline Poor quality & 0 & 0.0 & 32 & 74.4 & 30 & 90.9 & \multirow{3}{*}{$\begin{array}{c}\chi^{2}= \\
114.585^{*}\end{array}$} & \multirow{3}{*}{${ }^{\mathrm{MC}} \mathrm{p}<0.001^{*}$} \\
\hline Fair quality & 1 & 2.9 & 11 & 25.6 & 0 & 0.0 & & \\
\hline Good & 33 & 97.1 & 0 & 0.0 & 3 & 9.1 & & \\
\hline Mean \pm SD. & \multicolumn{2}{|c|}{$97.35 \pm 9.71$} & \multicolumn{2}{|c|}{$31.40 \pm 11.04$} & \multicolumn{2}{|c|}{$29.85 \pm 20.75$} & $\mathrm{~F}=253.689^{*}$ & $<0.001^{*}$ \\
\hline \multicolumn{9}{|l|}{ Psychological status } \\
\hline Poor quality & 0 & 0.0 & 23 & 53.5 & 14 & 42.4 & & \\
\hline Fair quality & 1 & 2.9 & 0 & 0.0 & 19 & 57.6 & & $<0.001^{*}$ \\
\hline Good & 33 & 97.1 & 20 & 46.5 & 0 & 0.0 & & \\
\hline Mean \pm SD & 97.6 & & 55. & 2.59 & 41. & & $\mathrm{~F}=98.308^{\square *}$ & $<0.001^{*}$ \\
\hline Working conditions & & & & & & & & \\
\hline Poor quality & 0 & 0.0 & 32 & 74.4 & 26 & 78.8 & & \\
\hline Fair quality & 0 & 0.0 & 11 & 25.6 & 7 & 21.2 & & $<0.001^{*}$ \\
\hline Good & 34 & 100.0 & 0 & 0.0 & 0 & 0.0 & & \\
\hline Mean \pm SD & 97.7 & & 24. & 8.49 & 20. & & $\mathrm{~F}=273.980^{*}$ & $<0.001^{*}$ \\
\hline Financial status & & & & & & & & \\
\hline Poor quality & 33 & 97.1 & 43 & 100.0 & 33 & 100.0 & & \\
\hline Fair quality & 1 & 2.9 & 0 & 0.0 & 0 & 0.0 & $x=$ & 0.608 \\
\hline Good & 0 & 0.0 & 0 & 0.0 & 0 & 0.0 & & \\
\hline Mean \pm SD & 27.0 & & 13. & 1.21 & & & $\mathrm{~F}=15.983^{*}$ & $<0.001^{*}$ \\
\hline Overall & & & & & & & & \\
\hline Poor quality & 0 & 0.0 & 43 & 100.0 & 33 & 100.0 & & \\
\hline Fair quality & 4 & 11.8 & 0 & 0.0 & 0 & 0.0 & $\begin{aligned} & x= \\
& 121480^{*}\end{aligned}$ & ${ }^{\mathrm{MC}} \mathrm{p}<0.001^{*}$ \\
\hline Good & 30 & 88.2 & 0 & 0.0 & 0 & 0.0 & 121.489 & \\
\hline Mean \pm SD & 81.0 & & 36. & & 29. & & $\mathrm{~F}=444.416^{*}$ & $<0.001^{*}$ \\
\hline
\end{tabular}
$\chi^{2}$ : Chi square test
MC: Monte Carlo for Chi square test
F: F test (ANOVA)
*: Statistically significant at $\mathrm{p} \leq 0.05$

Table (4) demonstrates that there were statistical significant relationship between Psoriasis severity and QoL scores, as the majority of patients with moderate to severe psoriasis had poor QoL. Also, there were statistical significant relationships between QoL scores and the disease history, as the majority of patients with psoriasis for more than one year had poor QoL, while the majority of patients with psoriasis for less than one year had 
fair to good QoL. No significant relationships between QoL scores of the studied patients and the rest of sociodemographic and clinical characteristics.

Table (4) Relationship between QoL Scores of the studied patients and their socio-demographic and clinical characteristics.

\begin{tabular}{|c|c|c|c|c|c|c|c|c|}
\hline \multirow{3}{*}{$\begin{array}{ll}\text { socio-demographic } & \text { and } \\
\text { clinical characteristics } & \end{array}$} & \multicolumn{6}{|c|}{ QoL the studied Scores } & \multirow{3}{*}{$\chi^{2}$} & \multirow{3}{*}{${ }^{\mathrm{MC}} \mathbf{p}$} \\
\hline & \multicolumn{2}{|c|}{$\begin{array}{l}<50 \% \text { poor quality of } \\
\text { life }\end{array}$} & \multicolumn{2}{|c|}{$\begin{array}{c}\mathbf{5 0}<\mathbf{7 0 \%} \text { fair } \\
\text { quality of life }\end{array}$} & \multicolumn{2}{|c|}{$>=75 \%$ good } & & \\
\hline & No & $\%$ & No & $\%$ & No & $\%$ & & \\
\hline \multicolumn{9}{|l|}{ Residence } \\
\hline Urban & 58 & 76.3 & 3 & 75.0 & 22 & 73.3 & \multirow{2}{*}{0.347} & \multirow{2}{*}{0.918} \\
\hline Rural & 18 & 23.7 & 1 & 25.0 & 8 & 26.7 & & \\
\hline \multicolumn{9}{|l|}{ Age/year } \\
\hline $30>40$ & 15 & 19.7 & 0 & 0.0 & 7 & 23.3 & \multirow{3}{*}{3.434} & \multirow{3}{*}{0.458} \\
\hline $40>50$ & 11 & 14.5 & 2 & 50.0 & 4 & 13.3 & & \\
\hline $50-60$ & 50 & 65.8 & 2 & 50.0 & 19 & 63.3 & & \\
\hline \multicolumn{9}{|l|}{ Sex } \\
\hline Male & 45 & 59.2 & 0 & 0.0 & 16 & 53.3 & \multirow{2}{*}{5.139} & \multirow{2}{*}{0.063} \\
\hline Female & 31 & 40.8 & 4 & 100.0 & 30 & 100.0 & & \\
\hline \multicolumn{9}{|l|}{ Education level } \\
\hline Illiterate & 39 & 51.3 & 1 & 25.0 & 20 & 66.7 & \multirow{4}{*}{9.256} & \multirow{4}{*}{0.105} \\
\hline Read and write & 12 & 15.8 & 2 & 50.0 & 5 & 16.7 & & \\
\hline High school or less & 19 & 25.0 & 0 & 0.0 & 5 & 16.7 & & \\
\hline University education or more & 6 & 7.9 & 1 & 25.0 & 0 & 0.0 & & \\
\hline \multicolumn{9}{|l|}{ Occupation } \\
\hline Clerical/Technical & 4 & 5.3 & 1 & 25.0 & 4 & 13.3 & \multirow{4}{*}{8.475} & \multirow{4}{*}{0.183} \\
\hline Manual & 34 & 44.7 & 0 & 0.0 & 15 & 50.0 & & \\
\hline Unemployed & 36 & 47.4 & 3 & 75.0 & 11 & 36.7 & & \\
\hline Retired & 2 & 2.6 & 0 & 0.0 & 0 & 0.0 & & \\
\hline \multicolumn{9}{|l|}{ Social status } \\
\hline Single & 3 & 3.9 & 0 & 0.0 & 0 & 0.0 & & \\
\hline Married & 64 & 84.2 & 3 & 75.0 & 28 & 93.3 & 3.357 & 0.460 \\
\hline Widow & 9 & 11.8 & 1 & 25.0 & 2 & 6.7 & & \\
\hline Family type & & & & & & & & \\
\hline Nuclear & 67 & 88.2 & 4 & 100.0 & 27 & 90.0 & & \\
\hline Extended & 9 & 11.8 & 0 & 0.0 & 3 & 10.0 & $0.1 / 9$ & 1.000 \\
\hline $\begin{array}{l}\text { Monthly income (Egyptian } \\
\text { pounds) }\end{array}$ & & & & & & & & \\
\hline$<1000$ & 47 & 61.8 & 2 & 50.0 & 20 & 66.7 & & \\
\hline $1000-2000$ & 28 & 36.8 & 1 & 25.0 & 9 & 30.0 & 5.751 & 0.193 \\
\hline$>2000$ & 1 & 1.3 & 4 & 100.0 & 30 & 100.0 & & \\
\hline Psoriasis severity & & & & & & & & \\
\hline Mild & 0 & 0.0 & 4 & 100.0 & 30 & 100.0 & & \\
\hline Moderate & 43 & 56.6 & 0 & 0.0 & 0 & 0.0 & & $0.001^{*}$ \\
\hline Sever & 33 & 43.4 & 0 & 0.0 & 0 & 0.0 & 121.489 & \\
\hline Psoriasis area Covered & & & & & & & & \\
\hline Exposed & 32 & 42.1 & 0 & 0.0 & 16 & 53.3 & & \\
\hline Covered & 44 & 57.9 & 4 & 100.0 & 14 & 46.7 & 3.995 & 0.129 \\
\hline Psoriasis history & & & & & & & & \\
\hline$<1$ year & 20 & 26.3 & 4 & 100.0 & 30 & 100.0 & & $0001^{*}$ \\
\hline$>$ 1year & 56 & 73.7 & 0 & 0.0 & 0 & 0.0 & & 0.001 \\
\hline
\end{tabular}

$\chi^{2}:$ Chi square test

*: Statistically significant at $\mathrm{p} \leq 0.05$

Table (5) shows that all mild cases of psoriasis had poor appetite, however, most moderate and severe grades of psoriasis had both poor to fair appetite, and the proportion increased significantly with increased psoriasis severity $(\chi 2=68.723 \& \mathrm{P}<0.001)$. All mild grade cases of psoriasis had higher scores in avoid eating certain foods $(\chi 2=32.440 \& \mathrm{P}<0.001)$.

Concerning sleep criteria, the table illustrates that all mild grade cases of psoriasis had good quality of sleep contrary to moderate cases. The majority of severe cases who had poor sleep because of itching and pain, and this result was statistically significant $\left(\chi^{2}=77.227 \& \mathrm{P}<0.001\right)$. Day sleep was more often affected in severe cases of psoriasis (48.5\%) than in moderate (46.5\%) and mild cases (2.9\%), although the difference was statistically significant $\left(\chi^{2}=72.566 \& \mathrm{P}<0.001\right)$.

In general, mild cases of psoriasis had good score in all physical activity items (swimming, walking for long distance, housekeeping, out of door, and selecting clothes), but moderate and severe case were extremely affected in all physical activity items and this result was highly significant $\left(\chi^{2}=135.410 \& \mathrm{P}<0.001, \chi^{2}=\right.$ 
$40.694 \& \mathrm{P}<0.001, \chi^{2}=62.631 \& \mathrm{P}<0.001, \chi^{2}=110.0 \& \mathrm{P}<0.001$, and $\left.\chi^{2}=102.938 \& \mathrm{P}<0.001\right)$ respectively.

Table (5) Relationship between severity of psoriasis and patients' activities of daily living and physical activities QoL scores

\begin{tabular}{|c|c|c|c|c|c|c|c|c|}
\hline \multirow{3}{*}{$\begin{array}{l}\text { Patients' activities of daily living and } \\
\text { physical activities' QoL scores }\end{array}$} & \multicolumn{6}{|c|}{ Severity of psoriasis } & \multirow{3}{*}{$\chi^{2}$} & \multirow{3}{*}{$\mathbf{P}$} \\
\hline & \multicolumn{2}{|c|}{$\begin{array}{l}\text { Mild } \\
(\mathrm{n}=\mathbf{3 4})\end{array}$} & \multicolumn{2}{|c|}{$\begin{array}{l}\text { Moderate } \\
(\mathrm{n}=\mathbf{4 3})\end{array}$} & \multicolumn{2}{|c|}{$\begin{array}{l}\text { Severe } \\
(\mathbf{n}=\mathbf{3 3})\end{array}$} & & \\
\hline & No & $\%$ & No & $\%$ & No & $\%$ & & \\
\hline \multicolumn{9}{|c|}{ Patient's activities of daily livings (eating, sleep) } \\
\hline \multicolumn{9}{|l|}{\begin{tabular}{l|l} 
Appetite &
\end{tabular}} \\
\hline$<50 \%$ poor & 34 & 100.0 & 12 & 27.9 & 15 & 45.5 & \multirow{3}{*}{$68.723^{*}$} & \multirow{3}{*}{$<0.001^{*}$} \\
\hline $50<75 \%$ fair & 0 & 0.0 & 11 & 25.6 & 18 & 54.5 & & \\
\hline$\geq 75 \%$ good & 0 & 0.0 & 20 & 46.5 & 0 & 0.0 & & \\
\hline \multicolumn{9}{|l|}{ Avoid eating certain foods } \\
\hline$<50 \%$ poor & 0 & 0.0 & 12 & 27.9 & 21 & 63.6 & \multirow{3}{*}{$32.440^{*}$} & \multirow{3}{*}{$<0.001^{*}$} \\
\hline $50<75 \%$ fair & 0 & 0.0 & 0 & 0.0 & 0 & 0.0 & & \\
\hline$\geq 75 \%$ good & 34 & 100.0 & 31 & 72.1 & 12 & 36.4 & & \\
\hline \multicolumn{9}{|l|}{ Sleep problems due to itching \&pain } \\
\hline$<50 \%$ poor & 0 & 0.0 & 43 & 100.0 & 19 & 57.6 & \multirow{3}{*}{$77.227^{*}$} & \multirow{3}{*}{$<0.001^{*}$} \\
\hline $50<75 \%$ fair & 0 & 0.0 & 0 & 0.0 & 0 & 0.0 & & \\
\hline$\geq 75 \%$ good & 34 & 100.0 & 0 & 0.0 & 14 & 42.4 & & \\
\hline \multicolumn{9}{|l|}{ Day naps. } \\
\hline$<50 \%$ poor & 1 & 2.9 & 20 & 46.5 & 16 & 48.5 & \multirow{3}{*}{$72.566^{*}$} & \multirow{3}{*}{$<0.001^{*}$} \\
\hline $50<75 \%$ fair & 31 & 91.2 & 0 & 0.0 & 10 & 30.3 & & \\
\hline$\geq 75 \%$ good & 2 & 5.9 & 23 & 53.5 & 7 & 21.2 & & \\
\hline \multicolumn{9}{|l|}{ Physical activities } \\
\hline \multicolumn{9}{|l|}{ Summer activities (swimming) } \\
\hline$<50 \%$ poor & 1 & 2.9 & 23 & 53.5 & 33 & 100.0 & \multirow{3}{*}{$135.410^{*}$} & \multirow{3}{*}{$<0.001^{*}$} \\
\hline $50<75 \%$ fair & 0 & 0.0 & 20 & 46.5 & 0 & 0.0 & & \\
\hline$\geq 75 \%$ good & 33 & 97.1 & 0 & 0.0 & 0 & 0.0 & & \\
\hline Walking for long distances & & & & & & & & \\
\hline$<50 \%$ poor & 1 & 2.9 & 23 & 53.5 & 26 & 78.8 & & \\
\hline $50<75 \%$ fair & 0 & 0.0 & 0 & 0.0 & 0 & 0.0 & $40.694^{*}$ & $<0.001^{*}$ \\
\hline$\geq 75 \%$ good & 33 & 97.1 & 20 & 46.5 & 7 & 21.2 & & \\
\hline Housekeeping activities & & & & & & & & \\
\hline$<50 \%$ poor & 0 & 0.0 & 32 & 74.4 & 29 & 87.9 & & \\
\hline $50<75 \%$ fair & 0 & 0.0 & 0 & 0.0 & 0 & 0.0 & $62.631^{*}$ & $<0.001^{*}$ \\
\hline$\geq 75 \%$ good & 34 & 100.0 & 11 & 25.6 & 4 & 12.1 & & \\
\hline Out door activities & & & & & & & & \\
\hline$<50 \%$ poor & 0 & 0.0 & 43 & 100.0 & 33 & 100.0 & & \\
\hline $50<75 \%$ fair & 0 & 0.0 & 0 & 0.0 & 0 & 0.0 & $110.000^{*}$ & $<0.001^{*}$ \\
\hline$\geq 75 \%$ good & 34 & 100.0 & 0 & 0.0 & 0 & 0.0 & & \\
\hline Selecting of clothes & & & & & & & & \\
\hline$<50 \%$ poor & 1 & 2.9 & 43 & 100.0 & 30 & 90.9 & & \\
\hline $50<75 \%$ fair & 1 & 2.9 & 0 & 0.0 & 0 & 0.0 & $102.938^{*}$ & p \\
\hline$\geq 75 \%$ good & 32 & 94.1 & 0 & 0.0 & 3 & 9.1 & & $<0.001$ \\
\hline
\end{tabular}

$\chi^{2}$ : Chi square test MC: Monte Carlo for chi square test

*: Statistically significant at $\mathrm{p} \leq 0.05$

Table (6) depicts that all patients with mild psoriasis had good QoL and that the majority of patients with severe psoriasis had poor QoL in both social and psychological domains. Those results were statistically significant. Surprisingly, more than three quarters $(77.3 \%)$ of the studied patients didn't embarrassed during sexual activities

Table (6): Relationship between severity of psoriasis and patients' social relations and Psychological and sexual feeling QoL scores

\begin{tabular}{|c|c|c|c|c|c|c|c|c|}
\hline \multirow{3}{*}{$\begin{array}{l}\text { patients' social relations and Psychological } \\
\text { and sexual feeling QoL scores }\end{array}$} & \multicolumn{6}{|c|}{ Severity of psoriasis } & \multirow{3}{*}{$\chi^{2}$} & \multirow{3}{*}{$\mathbf{p}$} \\
\hline & \multicolumn{2}{|c|}{$\begin{array}{l}\text { Mild } \\
(n=34)\end{array}$} & \multicolumn{2}{|c|}{$\begin{array}{l}\text { Moderate } \\
(\mathrm{n}=\mathbf{4 3})\end{array}$} & \multicolumn{2}{|c|}{$\begin{array}{l}\text { Severe } \\
(\mathbf{n}=\mathbf{3 3})\end{array}$} & & \\
\hline & No & $\%$ & No & $\%$ & No & $\%$ & & \\
\hline \multicolumn{9}{|l|}{ Social relationships } \\
\hline \multicolumn{9}{|l|}{ - Psoriasis affects my social status generally } \\
\hline$<50 \%$ poor. & 0 & 0.0 & 32 & 74.4 & 26 & 78.8 & \multirow{3}{*}{$79.486^{*}$} & \multirow{3}{*}{${ }_{<0.001^{*}}^{\mathrm{MC}_{\mathrm{p}}}$} \\
\hline $50<75 \%$ fair & 0 & 0.0 & 0 & 0.0 & 4 & 12.1 & & \\
\hline$\geq 75 \%$ good & 34 & 100.0 & 11 & 25.6 & 3 & 9.1 & & \\
\hline \multicolumn{9}{|l|}{ I Prefer being way from my family } \\
\hline$<50 \%$ poor & 0 & 0.0 & 23 & 53.5 & 7 & 21.2 & \multirow{2}{*}{$36.007^{*}$} & \multirow{2}{*}{${ }^{\mathrm{MC}} \mathrm{p}<0.001^{*}$} \\
\hline $50<75 \%$ fair & 1 & 2.9 & 0 & 0.0 & 4 & 12.1 & & \\
\hline
\end{tabular}


Quality of life of patients with Psoriasis in Alexandria- Egypt

\begin{tabular}{|c|c|c|c|c|c|c|c|c|}
\hline$\geq 75 \%$ good & 33 & 97.1 & 20 & 46.5 & 22 & 66.7 & & \\
\hline \multicolumn{9}{|c|}{ Psoriasis affects my family role } \\
\hline$<50 \%$ poor & 0 & 0.0 & 32 & 74.4 & 30 & 90.9 & \multirow{3}{*}{$114.585^{*}$} & \multirow{3}{*}{${ }^{\mathrm{MC}} \mathrm{p}<0.001^{*}$} \\
\hline $50<75 \%$ fair & 1 & 2.9 & 11 & 25.6 & 0 & 0.0 & & \\
\hline$\geq 75 \%$ good & 33 & 97.1 & 0 & 0.0 & 3 & 9.1 & & \\
\hline \multicolumn{9}{|c|}{ Avoid Making friends and meeting people } \\
\hline$<50 \%$ poor & 0 & 0.0 & 32 & 74.4 & 30 & 90.9 & \multirow{3}{*}{$114.585^{*}$} & \multirow{3}{*}{${ }^{\mathrm{MC}} \mathrm{p}<0.001^{*}$} \\
\hline $50<75 \%$ fair & 1 & 2.9 & 11 & 25.6 & 0 & 0.0 & & \\
\hline$\geq 75 \%$ good & 33 & 97.1 & 0 & 0.0 & 3 & 9.1 & & \\
\hline \multicolumn{9}{|c|}{ Worry about reactions of others } \\
\hline$<50 \%$ poor & 1 & 2.9 & 43 & 100.0 & 30 & 90.9 & \multirow{3}{*}{$93.204^{*}$} & \multirow{3}{*}{$<0.001^{*}$} \\
\hline $50<75 \%$ fair & 0 & 0.0 & 0 & 0.0 & 0 & 0.0 & & \\
\hline$\geq 75 \%$ good & 33 & 97.1 & 0 & 0.0 & 3 & 9.1 & & \\
\hline \multicolumn{9}{|c|}{ Psychological and sexual feelings } \\
\hline \multicolumn{9}{|c|}{ Feel embarrassed } \\
\hline$<50 \%$ poor. & 1 & 2.9 & 23 & 53.5 & 11 & 33.3 & \multirow{3}{*}{$85.337^{*}$} & \multirow{3}{*}{$<0.001^{*}$} \\
\hline $50<75 \%$ fair & 0 & 0.0 & 0 & 0.0 & 19 & 57.6 & & \\
\hline$\geq 75 \%$ good & 33 & 97.1 & 20 & 46.5 & 3 & 9.1 & & \\
\hline \multicolumn{9}{|c|}{ Feel short tempered } \\
\hline$<50 \%$ poor & 1 & 2.9 & 23 & 53.5 & 7 & 21.2 & \multirow{3}{*}{$102.532^{*}$} & \multirow{3}{*}{$<0.001^{*}$} \\
\hline $50<75 \%$ fair & 0 & 0.0 & 20 & 46.5 & 23 & 69.7 & & \\
\hline$\geq 75 \%$ good & 33 & 97.1 & 0 & 0.0 & 3 & 9.1 & & \\
\hline \multicolumn{9}{|c|}{ I have no body to talk } \\
\hline$<50 \%$ poor & 0 & 0.0 & 23 & 53.5 & 6 & 18.2 & \multirow{3}{*}{$33.901^{*}$} & \multirow{3}{*}{${ }^{\mathrm{MC}} \mathrm{p}<0.001^{*}$} \\
\hline $50<75 \%$ fair & 1 & 2.9 & 0 & 0.0 & 0 & 0.0 & & \\
\hline$\geq 75 \%$ good & 33 & 97.1 & 20 & 46.5 & 27 & 81.8 & & \\
\hline \multicolumn{9}{|l|}{ Feel hopeless } \\
\hline$<50 \%$ poor & 0 & 0.0 & 23 & 53.5 & 33 & 100.0 & \multirow{3}{*}{$85.736^{*}$} & \multirow{3}{*}{${ }^{\mathrm{MC}} \mathrm{p}<0.001^{*}$} \\
\hline $50<75 \%$ fair & 1 & 2.9 & 0 & 0.0 & 0 & 0.0 & & \\
\hline$\geq 75 \%$ good & 33 & 97.1 & 20 & 46.5 & 0 & 0.0 & & \\
\hline \multicolumn{9}{|c|}{ Feeling of stigma } \\
\hline$<50 \%$ poor. & 0 & 0.0 & 43 & 100.0 & 30 & 90.9 & \multirow{3}{*}{$123.719^{*}$} & \multirow{3}{*}{${ }^{\mathrm{MC}} \mathrm{p}<0.001^{*}$} \\
\hline $50<75 \%$ fair & 1 & 2.9 & 0 & 0.0 & 3 & 9.1 & & \\
\hline$\geq 75 \%$ good & 33 & 97.1 & 0 & 0.0 & 0 & 0.0 & & \\
\hline \multicolumn{9}{|c|}{$\begin{array}{l}\text { Feel embarrassed during sexual relations (not } \\
\text { applicable = 15) }\end{array}$} \\
\hline$<50 \%$ poor & 0 & 0.0 & 0 & 0.0 & 0 & 0.0 & & \\
\hline $50<75 \%$ fair . & 1 & 3.2 & 0 & 0.0 & 5 & 22.7 & $10.211^{*}$ & ${ }^{\mathrm{MC}} \mathrm{p} 0.002^{*}$ \\
\hline$\geq 75 \%$ good. & 30 & 96.8 & 42 & 100.0 & 17 & 77.3 & & \\
\hline
\end{tabular}

$\chi^{2}$ : Chi square test $\quad$ MC: Monte Carlo for chi square test

*: Statistically significant at $\mathrm{p} \leq 0.05$

Table (7) demonstrates that there were significant relationships between QoL of patients' working conditions and the severity of the disease, since the majority of patients with mild psoriasis had good working conditions, while the majority with severe cases had poor working conditions $\left(\chi^{2}=110.285^{*}, \mathrm{p}<0.001\right)$ and $\left(\chi^{2}=105.838^{*}, \mathrm{p}\right.$ $<0.001)$ respectively. Also, there were significant relationships between QoL of patients' financial status and the severity of the disease, as all patients with severe psoriasis, had poor QoL since they couldn't afford treatment costs and had no health insurance.

Table (7): Relationship between severity of psoriasis with patients' working conditions and financial status QoL scores

\begin{tabular}{|c|c|c|c|c|c|c|c|c|}
\hline \multirow{3}{*}{$\begin{array}{l}\text { patients' working conditions and financial } \\
\text { status QoL scores }\end{array}$} & \multicolumn{6}{|c|}{ Severity of psoriasis } & \multirow{3}{*}{$\chi^{2}$} & \multirow{3}{*}{$\mathbf{p}$} \\
\hline & \multicolumn{2}{|c|}{$\begin{array}{l}\text { Mild } \\
(n=34)\end{array}$} & \multicolumn{2}{|c|}{$\begin{array}{l}\text { Moderate } \\
(\mathrm{n}=43)\end{array}$} & \multicolumn{2}{|c|}{$\begin{array}{l}\text { Severe } \\
(\mathbf{n}=\mathbf{3 3})\end{array}$} & & \\
\hline & No & $\%$ & No & $\%$ & No & $\%$ & & \\
\hline \multicolumn{9}{|l|}{ Working conditions } \\
\hline \multicolumn{9}{|l|}{ Psoriasis oppose my working conditions } \\
\hline$<50 \%$ poor & 0 & 0.0 & 32 & 74.4 & 26 & 78.8 & \multirow{3}{*}{$110.285^{*}$} & \multirow{3}{*}{$<0.001^{*}$} \\
\hline $50<75 \%$ fair & 0 & 0.0 & 11 & 25.6 & 7 & 21.2 & & \\
\hline$\geq 75 \%$ good & 34 & 100.0 & 0 & 0.0 & 0 & 0.0 & & \\
\hline \multicolumn{9}{|l|}{ My performance affected by psoriasis } \\
\hline$<50 \%$ poor & 0 & 0.0 & 32 & 74.4 & 26 & 78.8 & \multirow{3}{*}{$105.838^{*}$} & \multirow{3}{*}{$<0.001^{*}$} \\
\hline $50<75 \%$ fair & 1 & 2.9 & 11 & 25.6 & 7 & 21.2 & & \\
\hline$\geq 75 \%$ good & 33 & 97.1 & 0 & 0.0 & 0 & 0.0 & & \\
\hline \multicolumn{9}{|l|}{ Financial status } \\
\hline \multicolumn{9}{|l|}{ I can't afford treatment costs } \\
\hline$<50 \%$ poor & 3 & 8.8 & 43 & 100.0 & 33 & 100.0 & \multirow{2}{*}{$96.485^{*}$} & \multirow{2}{*}{$<0.001^{*}$} \\
\hline $50<75 \%$ fair & 31 & 91.2 & 0 & 0.0 & 0 & 0.0 & & \\
\hline
\end{tabular}


Quality of life of patients with Psoriasis in Alexandria- Egypt

\begin{tabular}{|c|c|c|c|c|c|c|c|c|}
\hline$\geq 75 \%$ good & 0 & 0.0 & 0 & 0.0 & 0 & 0.0 & & \\
\hline \multicolumn{9}{|c|}{ Hospital is away from home } \\
\hline$<50 \%$ poor & 27 & 79.4 & 23 & 53.5 & 9 & 27.3 & \multirow{3}{*}{$74.470^{*}$} & \multirow{3}{*}{$<0.001^{*}$} \\
\hline $50<75 \%$ fair & 4 & 11.8 & 20 & 46.5 & 0 & 0.0 & & \\
\hline$\geq 75 \%$ good & 3 & 8.8 & 0 & 0.0 & 24 & 72.7 & & \\
\hline \multicolumn{9}{|c|}{ Have no health insurance } \\
\hline$<50 \%$ poor & 34 & 100.0 & 23 & 53.5 & 33 & 100.0 & \multirow{3}{*}{$38.088^{*}$} & \multirow{3}{*}{$<0.001^{*}$} \\
\hline $50<75 \%$ fair & 0 & 0.0 & 20 & 46.5 & 0 & 0.0 & & \\
\hline$\geq 75 \%$ good & 0 & 0.0 & 0 & 0.0 & 0 & 0.0 & & \\
\hline \multicolumn{9}{|c|}{$\begin{array}{l}\text { Treatment negatively affects } \text { other } \\
\text { financial requirements }\end{array}$} \\
\hline$<50 \%$ poor & 4 & 11.8 & 43 & 100.0 & 33 & 100.0 & \multirow{3}{*}{$94.363^{*}$} & \multirow{3}{*}{$\begin{array}{c}{ }^{\mathrm{MC}} \mathrm{p} \\
<0.001\end{array}$} \\
\hline $50<75 \%$ fair & 28 & 82.4 & 0 & 0.0 & 0 & 0.0 & & \\
\hline$\geq 75 \%$ good & 2 & 5.9 & 0 & 0.0 & 0 & 0.0 & & \\
\hline
\end{tabular}

$\chi^{2}:$ Chi square test

$*$ : Statistically significant at $\mathrm{p} \leq 0.05$

\section{Discussion:}

Psoriasis is a serious dermatological disease extending beyond the physical symptoms experienced by the patient, and seriously affecting the way in which the patient sees him /herself and the way he/she is seen by others. The main findings revealed by the current study were the negative impact of psoriasis on the majority of Psoriasis patients' quality of life and the positive association between disease severity and patients' poor quality of life. Supporting our results, Głowacka et al (2014) illustrated that Psoriasis had its negative effect on patients' QoL ${ }^{(30)}$. In addition, Barot et al (2015) reported in a similar study that the majority of psoriasis patients had moderate to large negative impact on their $\mathrm{QLL}^{(31)}$.

Results of the present study found that patients with moderate to severe psoriasis had poor to fair QoL, while patients with mild psoriasis had good QoL in all domains except the financial, which was poor for all levels of disease severity, nevertheless these results were insignificant. These findings were comparable to previous studies, which established a good correlations between disease severity and $\mathrm{QoL}^{(32-36)}$.

However, the current study findings differ from those findings of Perira da Silva et al (2013) who mentioned that severity of the disease as evaluated by health practitioners was not associated with impaired QoL reported by the patients ${ }^{(37)}$. The authors concluded that the impact of psoriasis on QoL is not always correlated with clinical severity, as mild lesions may have severe psychosocial problems ${ }^{(38)}$.

Moreover, the current study indicated that the majority of the studied patients had poor QoL in relation to physical activity, social relationships, and some items of psychosocial status. Similarly, Mazeedi et al (2006) found that living with psoriasis affect patients' physical, psychological, social functioning, and well-being, and that moderate to severe cases were more affected than mild cases ${ }^{(39)}$. In this regard, Gelfand et al (2004) and EL-Moselhy et al (2105) cleared that patients with severe psoriasis had higher rates of physical and psychosocial disabilities which confirming the results of previous studies ${ }^{(40,41)}$. Hrehorów et al (2007), also illustrated that itching and pain had made physical activities difficult task for patients with psoriasis ${ }^{(42)}$.

Psoriasis affects patients' role in the family, hinders them from meeting people, and increase their worry about reactions of others. The present study demonstrated significant negative association between Psoriasis severity and social relationships. This finding is consistent with Bronikowska-Kolasa (2008) ${ }^{(43)}$. In this context, Bhosle et al (2006) reported that the majority of their studied patients, were impressed by the way they are seen by others who observe their lesions ${ }^{(44)}$.

As regards psychological feelings, the results showed that all patients with severe psoriasis had poor QoL regarding feeling lack of hope and stigma. This could be related to the disfigurement effect of psoriasis since it is a visible disease. In the same line, Kirby et al (2001) and, Fortune et al (2005) mentioned that feelings of stigma, and shame regarding their appearance are frequent in patients with Psoriasis ${ }^{(45,46)}$.

Surprisingly, the majority of the studied patients didn't feel embarrassed during sexual activities regardless severity of the disease. Contrary to our results, Mazeedi et al (2006) found significant associations between disease severity and sexual activity as patients with severe psoriasis were more affected than those with mild and moderate psoriasis ${ }^{(39)}$. Also, Głowacka et al (2014) illustrated that psoriasis limit patients' sexual life because of their embarrassment ${ }^{(37)}$.

The current study also demonstrated that most moderate and severe cases of psoriasis either had poor or fair appetite, and the proportion increases significantly with increased Psoriasis severity. For example, all mild cases of Psoriasis had higher score in avoid eating certain foods than moderate to severe cases. This probably related to itching, pain, and poor sleeping hours reported by patients. Those results are coherent with the findings of Khwaja et al (2015) who found alteration in dietary habits associated with disease severity ${ }^{(47)}$. In addition, high statistical significant association was found between severity of the disease and sleep. This could 
be related to itching, pain reported by the studied patients. Similar findings were reported by Głowacka et al $(2014)^{(30)}$. This is also consistent with previous studies ${ }^{(48,49)}$.

Absenteeism is a greater concern for people suffering from psoriasis ${ }^{(44)}$. Significantly in the current study, QoL related to patients' working conditions was negatively associated with severity of the disease, since the majority of patients with severe cases had poor working conditions. This could be attributed to the missing work days due to treatment schedule or Psoriasis complications as reported by the study subjects. In this context, Milcic et al (2015) mentioned that unemployment was significantly correlated with severity of psoriasis ${ }^{(50)}$. Also, many studies had reported a high proportion of reduction in work productivity and sick leaves in patients with severe disease which added a greater financial burden ${ }^{(51,52,53)}$. In this regard, the present study revealed a significant negative association between severity of psoriasis and patients' financial status, as almost all patients with severe psoriasis couldn't afford treatment costs and had no health insurance coverage. Financial burden to the patient and family and its interference with work, a decrease in QoL, and money matters was reported in previous studies ${ }^{(54-56)}$.

However, our findings revealed that there were no statistical associations between psoriasis severity and patients' age or sex. Also, Ross (1997) did not find any significant association between patients age, sex, and QoL scores ${ }^{(57)}$. In this regard, Głowacka et al (2014) illustrated that age played insignificant role, since there was no significant difference between older and younger patients and the severity of the disease ${ }^{(30)}$. However, Zachariae et al (2002) reported that older patients had better QoL compared with the younger ${ }^{(58)}$. Sampogna et al (2006) mentioned that older patients with psoriasis had poorer life $\mathrm{QOL}^{(59)}$. This difference could mean that the impact of age factor on the QoL of patients with psoriasis may vary among patients and should be studied. On the other hand, Głowacka et al (2014) found that Women have poorer quality of life than men since women are overwhelmed with their appearance ${ }^{(30)}$.

According to the current study results, there were insignificant associations between QoL scores and patient's education, or involvement of visible area. Contrary to our results, Ross (1997) found that patients with higher education had good quality of life as education increase their knowledge, and allow them to manage themselves better ${ }^{(57)}$. Touw et al (2001) found that involvement of visible areas of the body causes more negative impact on patients QoL than involvement of invisible areas ${ }^{(60)}$.

There was a statistical significant relationship between QoL scores and the disease history, as the majority of patients with psoriasis for more than one year had poorer QoL, while the majority of patients with psoriasis for less than one year had fair to good QoL. On the contrary, Ross et al (1997) did not find any significant association between duration of the disease, and quality of life ${ }^{(57)}$.

Nurses have a unique role in patient education, and planning nursing interventions aiming at minimizing the consequences of psoriasis, and improving patients' quality of life through effective management. They also have the responsibility of encouraging patients to comply with their treatment and suggest lifestyle modifications that will potentially improve patients' quality of life.

\section{Conclusion}

In conclusion, our results document that psoriasis exerts significant, negative impact on patients' quality of life. The majority of the studied patients had poor QoL in relation to physical activity, social relationships, working conditions, and financial status, fair QoL in relation to activities of daily living as eating and sleeping, and good QoL in relation to psychosocial and sexual health. Results highlighted strong statistically significant association between Psoriasis severity and some demographic and clinical factors as patient's education, social status, and having the disease for more than one year. In addition, high statistical significant associations were found between severity of psoriasis and all domains of patients' QoL. Patients with moderate to severe psoriasis had poor to fair QoL, while patients with mild psoriasis had good QoL in all domains except the financial one, which was poor.

\section{Recommendations}

- Studies including both the private and governmental sectors of patients with psoriasis should be implemented.

- More research should be carried out in different areas in Egypt, to understand the impact of psoriasis on patients' QoL.

- Standard of care should be developed to improve the psoriasis patients' QoL

- Develop rehabilitation programs to enhance psoriasis patient's self management and QoL.

- Establishment of a hot line contact for patients' counseling is required

\section{References}

[1]. Feldman SR. Epidemiology, pathophysiology, clinical manifestations, and diagnosis of psoriasis. In: Basow DS, editor. Up-to-date. Waltham, MA: Up-to-date Inc; 2011. 
[2]. Naldi L. Epidemiology of psoriasis. Curr Drug Targets Inflamm Allergy. 2004;3:121-128.

[3]. Harden JL, Krueger JG, Bowcock AM. The immunogenetics of psoriasis: a comprehensive review. J Autoimmun. 2015;64:66-73.

[4]. Boehncke W-H, Schon MP. Psoriasis. Lancet. 2015;386(9997):983-94.

[5]. WHO Global report on psoriasis. WHO 2016. http://apps.who.int/iris/bitstream/10665/204417/1/9789241565189 eng.pdf. Retrieved on: 9/12/2016.

[6]. Danielsen K, Olsen AO, Wilsgaard T, Furberg AS. Is the prevalence of psoriasis increasing? A 30-year follow-up of a populationbased cohort. Br J Dermatol. 2013;168:1303-10.

[7]. Lebwohl M. Psoriasis. Lancet. 2003;361:1197-1204.

[8]. Stern RS, Nijsten T, Feldman SR, Margolis DJ, Rolstad T. Psoriasisis common, carries a substantial burden even when not extensive, and is associated with widespread treatment dissatisfaction. J Investig Dermatol Symp Proc. 2004;9:136-139.

[9]. Parisi R, Symmons DPM, Griffiths CEM, Ashcroft DM, and the Identification and Management of Psoriasis and Associated ComorbidiTy (IMPACT) project team. Global epidemiology of psoriasis: a systematic review of incidence and prevalence. J Invest Dermatol. 2013;133(2):377-85.

[10]. Pietrangelo A. Psoriasis by numbers: Facts, Statistics 2015 Available @ http://www.healthline.com/health/psoriasis/facts-statisticsinfographic.retrieved on: 9/9/2015.

[11]. Fuji R, Mould JF J, Tang B, Brandt H, Pomerantz D, Chapnick J et al. Burden of disease in patients with diagnosed psoriasis in Brazil: results from 2011 national health and wellness survey (NHWS). Value Health. 2012;15(4):A107.

[12]. De Korte J, Sprangers MA, Mombers FM, Bos JD. Quality of life in patients with psoriasis: a systematic literature review. J Investig Dermatol Symp Proc. 2004;9(2):140-7.

[13]. Kimball AB, Gieler U, Linder D, Sampogna F, Warren RB, Augustin M. Psoriasis: Is the impairment to a patient's life cumulative? J Eur Acad Dermatol Venereol. 2010;24(9):989-1004.

[14]. Moradi M, Rencz F, Brodszky V, Moradi A, Balogh O, Gulacsi L. Health status and quality of life in patients with psoriasis: an Iranian cross-sectional survey. Arch Iran Med. 2015;18(3):153-9.

[15]. Tang MM, Chang CC, Chan LC, Heng A. Quality of life and cost of illness in patients with psoriasis in Malaysia: amulticenter study. Int J Dermatol. 2013;52(3):314-22.

[16]. Augustin M, Kruger K, Radtke MA, Schwippl I, Reich K. Disease severity, quality of life and health care in plaque type psoriasis: a multicenter cross-sectional study in Germany. Dermatology. 2008;216(4):366-72.

[17]. Sampogna F, Tabolli S, Abeni D, IDI Multipurpose Psoriasis Research on Vital Experiences (IMPROVE) investigators. Living with psoriasis: prevalence of shame, anger, worry, and problems in daily activities and social life. Acta Derm Venereol. 2012;92(3):299303.

[18]. Russo PAJ, Ilchef R, Cooper AJ. Psychiatric morbidity in psoriasis: a review. Australas J Dermatol. 2004;45(3):155-9; quiz;160-1.

[19]. Kimball AB, Jacobson C, Weiss S, Vreeland MG, Wu Y. The psychosocial burden of psoriasis. Am J Clin Dermatol. 2005;6(6):383-92.

[20]. Zachariae H, Zachariae R, Blomqvist K, Davidsson S, Molin L, Mork C et al. Quality of life and prevalence of arthritis reported by 5,795 members of the Nordic Psoriasis Associations. Data from the Nordic Quality of Life Study. Acta Derm Venereol. 2002;82(2):108-13.

[21]. Krueger G, Koo J, Lebwohl M, Menter A, Stern RS, Rolstad T. The impact of psoriasis on quality of life: results of a 1998 National Psoriasis Foundation patient-membership survey. Arch Dermatol. 001;137(3):280-4.

[22]. Weiss SC, Kimball AB, Liewehr DJ, Blauvelt A, Turner ML, Emanuel EJ. Quantifying the harmful effect of psoriasis on healthrelated quality of life. J Am Acad Dermatol. 2002;47(4):512-8.

[23]. Pereira MG, Brito L, Smith T. Dyadic adjustment, family coping, body image, quality of life and psychological morbidity in patients with psoriasis and their partners. Int J Behav Med. 2012;19(3):260-9.

[24]. Berger K, Ehlken B, Kugland B, Augustin M. Cost-of-illness in patients with moderate and severe chronic psoriasis vulgaris in Germany. J Dtsch Dermatol Ges. 2005;3(7):511-8.

[25]. Gaikwad R, Deshpande S, Raje S, Dhamdhere DV, Ghate MR. Evaluation of functional impairment in psoriasis. Indian J Dermatol Venereol Leprol. 2006;72(1):37-40.

[26]. Sohn S, Schoeffski O, Prinz J, Reich K, Schubert E, Waldorf K et al. Cost of moderate to severe plaque psoriasis in Germany: a multicenter cost-of-illness study. Dermatology. 2006;212(2):137-44.

[27]. Brown I, Brown R. Quality of Life and Disability: An Approach for community Practitioners. London: Jessica Kingsley; 2004(2):17-27.

[28]. Stefan C. Weiss, Kendra G. Bergstrom, Sally A. Weiss, Alexa B. Kimball. Quality of Life Considerations in Psoriasis Treatment. J Dermatol Nursing. 2003;15(2)

[29]. Morgan M, Mc Creedy R, Simpson J, Hay RJ. Dermatology quality of life scales-a measure of the impact of skin diseases. Br J Dermatol. $1997 \mathrm{Feb}$;136(2):202-6

[30]. Głowacka M, Kornatowska K, Kempińska A, and, Lishchynskyy Y. Quality of life in patients with psoriasis as a determinant of nursing care. JBHNMR; 2014(2): 32- 8 .

[31]. Barot P, Brahmbhatt N, Ninama H, Kharadi D, and, Malhotra S. Quality of life in patients with psoriasis at a tertiary care teaching hospital -a cross sectional study. NJMR | Volume 5 | Issue 2 | Apr - Jun 2015 PG: 93-7.

[32]. Reich K, Griffiths CE. The relationship between quality of life and skin clearance in moderate-to-severe psoriasis: lessons learnt from clinical trials with infliximab. Arch Dermatol Res. 2008;300:537-44.

[33]. Mazzotti E, Picardi A, Sampogna F, Sera F, Pasquini P, Abeni D, et al. Sensitivity of the Dermatology Life Quality Index to clinical change in patients with psoriasis. Br J Dermatol. 2003;149:318-22.

[34]. Rakhesh SV, D'Souza M, Sahai A. Quality of life in psoriasis: a study from south India. Indian J Dermatol Venereol Leprol. 2008;74:600-6.

[35]. Revicki DA, Willian MK, Menter A, Saurat JH, Harnam N, Kaul M. Relationship between clinical response to therapy and healthrelated quality of life outcomes in patients with moderate to severe plaque psoriasis. Dermatology. 2008;216:260-70.

[36]. Gelfand JM, Feldman SR, Stern RS, Thomas J, Rolstad T, Margolis DJ. Determinants of quality of life in patients with psoriasis: a study from the US population. J Am Acad Dermatol. 2004;51:704-8.

[37]. Pereira da Silva M, Bartoli Miot L, Fortes M, Marques S. Psoriasis: correlation between severity index (PASI) and quality of life index (DLQI) in patients assessed before and after systemic treatment An Bras Dermatol. 2013;88(5):760-3.

[38]. De Arruda LHF, de Moraes APF. The impact of psoriasis on quality of life. Br J Dermatol 2001; 144: 33-36.

[39]. Mazeedi K, El-Shazly M, Al-Ajmi H. Impact of psoriasis on quality of life in Kuwait. International Journal of Dermatology. 2006, $45,418-424$ 
[40]. Gelfand JM, Feldman SR, Stern RS, Thomas J, Rolstad T, et al. Determinants of quality of life in patients with psoriasis: a study from the US population. J Am Acad Dermatol. 2004, 51: 704-708.

[41]. El-Moselhy E, Atlam S, Mohammed A, Hassan H, Ebrahim A. Psychosocial Impact and Quality of Life among Adult Egyptian Patients with Psoriais. Dermatol Case Rep 2015,1:1

[42]. Hrehorów E, Reich A, Szepietowski J. Jakość życia chorych na łuszczycę; zależność od świądu, stresu i objawów depresyjnych. Derm Klin; 2007, 9(1); 19-23.

[43]. Bronikowska - Kołasa A, Borzęcki A, Szponar A, i wsp. Ocena wybranych aspektów jakości życia pa-cjentów z łuszczyca za pomocą kwestionariusza WHOQOL -100. Nowa Med; 2008, 3: 4-11. In Głowacka M, Kornatowska K, Kempińska A, and, Lishchynskyy Y.uality of life in patients with psoriasis as a determinant of nursing care. JPHNMR; 2014 (2): 32 - 8.

[44]. Bhosle M, Kulkarni A, Feldman S, and Balkrishnan R. Quality of life in patients with psoriasis. Health and Quality of Life Outcomes 2006, 4:35 pg: 1-7.

[45]. Kirby B, Richards HL, Hindle E, et al. Physical and psychological measures are necessary to assess overall psoriasis severity. J Am Acad Dermatol 2001; 45: 72-76.

[46]. Fortune DG, Richards HL, Griffiths CE: Psychologic factors in psoriasis: consequences, mechanisms, and interventions. Dermatol Clin 2005, 23:681-694. In Bhosle M, Kulkarni A, Feldman S, and Balkrishnan R. Quality of life in patients with psoriasis. Health and Quality of Life Outcomes 2006, 4:35 pg: 1-7.

[47]. Khawaja A, Bokhari S, Rasheed T, Shahzad A, Hanif M, Qadeer F, Jafferany M. Disease Severity, Quality of Life, and Psychiatric Morbidity in Patients With Psoriasis With Reference to Sociodemographic, Lifestyle, and Clinical Variables: A Prospective, CrossSectional Study From Lahore, Pakistan. Prim Care Companion CNS Disord. 2015; 17(3): 10

[48]. Dhamdhere V, Gaikwad R, Deshpande S, Raje S, Dhamdhere D, GhateM, Evaluation of functional impairment in psoriasis Evaluation of functional impairment in psoriasis. Indian J Dermatol Venereol Leprol 2006;72(1): 1

[49]. Sharma N, Koranne RV, Singh RK. Psychiatric morbidity in psoriasis and vitiligo-a comparative study. J Dermatol 2001;28:419-23

[50]. Milcic D, Jankovic S, Vesic S, Milinkovic S, and Jankovic J. Assessment of quality of life in patients with psoriasis: a study from Serbia. International Journal of Dermatology 2015, 54, 523-528.

[51]. Finlay AY, Coles EC: The effect of severe psoriasis on the quality of life of 369 patients. Br J Dermatol 1995, 132:236-244

[52]. Feldman SR, Fleischer AB Jr, Reboussin DM, Rapp SR, Bradham DD, Exum ML, Clark AR: The economic impact of psoriasis increases with psoriasis severity. J Am Acad Dermatol 1997, 37:564-569.

[53]. Tan X, Feldman S, and Balkrishnan R. Quality of life issues and measurement in patients with psoriasis. Psoriasis: Targets and Therapy 2012:2 13-23.

[54]. NPF (National Psoriasis Foundation) (2009) National psoriasis foundation report on the psycho-social impacts of psoriasis.

[55]. Choi J, Koo JY (2003) Quality of life issues in psoriasis. J Am Acad Dermatol 49: S57-61.

[56]. Wiklund I, Hanestad BR (2000) The burden of psoriasis: A study concerning health-related quality of life among Norwegian adult patients with psoriasis compared with general population norms. Qual Health Res 12: 250-61.

[57]. Ross CE. Education and the subjective quality of life. J Health Soc Behav 1997; 38: 275-297. In: Milcic D, Jankovic S, Vesic S, Milinkovic S, and Jankovic J. Assessment of quality of life in patients with psoriasis: a study from Serbia. International Journal of Dermatology 2015; 54:523-28.

[58]. Zachariae R, Zachariae H, Blomqvist K, Davidsson S, Molin L, Mork C et al. Quality of life in 6497 Nordic patients with psoriasis. Br J Dermatol 2002; 146: 1006-16. In: Barot P, Brahmbhatt N, Ninama H, Kharadi D, and, Malhotra S. Quality of life in patients with psoriasis at a tertiary care teaching hospital -a cross sectional study. NJMR | Volume 5 | Issue 2||Apr - Jun 2015 PG: 93-7

[59]. Sampogna F, Chren MM, Melchi CF, Pasquini P, Tabolli S, Abeni D. Age, gender, quality of life and psychological distress in patients hospitalized with psoriasis. Br J Dermatol 2006; 154:325-31. In: Barot P, Brahmbhatt N, Ninama H, Kharadi D, and, Malhotra S. Quality of life in patients with psoriasis at a tertiary care teaching hospital -a cross sectional study. NJMR/Volume 5 | Issue 2 | Apr - Jun 2015 PG: 93-7.

[60]. Touw C, Roijen L, Verboom P, Paul C, Finaly A. Quality of life and clinical outcome in psoriasis patients using intermittent cyclosporine. British Journal of Dermatology 2001; 144: 967-72. 